\title{
Comparison of four non-Bayesian methods to estimate the scale parameter for Modified Weibull distribution by using the Simulation
}

\author{
Iden Hassan Hussein \\ University of Baghdad \\ College of Science for Women \\ Idenalkanani@yahoo.com
}

\author{
Hazim Ghdhaib Kalt \\ University of Karbala \\ College of Education for pure science \\ Hazim.galit@uokerbala.edu.iq
}

Recived : $16 \backslash 4 \backslash 2018$

Revised : 25\4\2018

Accepted : $26 \backslash 4 \backslash 2018$

Available online : 3 /6/2018

DOI: $10.29304 / j q c m .2018 .10 .2 .393$

\begin{abstract}
.,
In this paper, four methods were obtained to estimate the scale parameter of Modified Weibull distribution using complete data, which are the Modified Moments Estimator (MME), the Maximum Likelihood Estimator (MLE), White Estimator (WE) and Least Squares Estimator (LSE), Monte Carlo simulation is used to compare these four estimators with respect to the Mean Square Error criteria (MSE), and the results on simulated samples of the comparison showed that for all the varying sample size in this study, and in all cases for the four methods The MLE method is best followed by the OLSE method then the WE method and the MME method .
\end{abstract}

Key Words. Modified Weibull distribution, Modified Moments Estimator, Least Squares Estimator, White Estimator.

\section{Introduction}

The probability density function of any random variable $\mathrm{t}$ having a modified Weibull distribution (MWD) with scale parameter $\alpha>0$ and both shape parameters $\beta \geq 0$ and $\lambda>0$ is given by

$f(t ; \alpha, \beta, \lambda)= \begin{cases}\alpha(\beta+\lambda t) t^{\beta-1} \exp \left(\lambda t-\alpha t^{\beta} e^{\lambda t}\right) & , t \geq 0(1) \\ 0 & , \text { ow }\end{cases}$

If $\beta=0$, the resulting distribution is called the type 1 extreme-value which is also known as a log-gamma distribution when $\lambda=0$ then MWD reduces to the two-parameter Weibull distribution. Also when both $\beta=2$ and $\lambda=0$ then MWD reduces to oneparameter Rayleigh distribution. The modified Weibull model was developed by Xie et al.(2003) [1] this lifetime distribution is an important feature for reliability analysis.
Vasile et al. [2] studied the method of Bayes to estimate the parameters of the MWD and Upadhyaya and Gupta [3] using Markov chain Monte Carlo simulation to study the Bayes analysis of the MWD. Ateya [4] study the estimation problem of the censored sample of order statistics generalized from MWD.

The cumulative distribution function and reliability function respectively are

$F(t ; \alpha, \beta, \lambda)=1-\exp \left(-\alpha t^{\beta} e^{\lambda t}\right)$

$R(t)=\exp \left(-\alpha t^{\beta} e^{\lambda t}\right)$

We review four methods which are the Modified Moments Estimator (MME), the Maximum Likelihood Estimator (MLE), White Estimator (WE) and Least Squares Estimator (LSE), These methods are compared in Section 6, using the mean square error (MSE) criteria, all these four methods which are using to estimate the scale parameter for Modified Weibull are non-Bayesian methods . 
Iden .H/Hazim .G

\section{Modified Moments Estimator (MME)}

(Whitten and Cohen), in (1982), proposed a new modification on moment method [5], using the following equation

$$
\begin{aligned}
& E\left(\hat{F}\left(t_{(i)}\right)\right)=F\left(t_{(i)}\right) \quad i=1,2, \ldots, n \\
& t_{(1)} \leq t_{(2)} \leq . \quad . \quad . \leq t_{(n)}
\end{aligned}
$$

Where $t_{(i)}$ is the i's order random variable, $\hat{F}\left(t_{(i)}\right)$ is estimated unbiased for function distribution $F\left(t_{(i)}\right)$ and by replacement $F\left(t_{(i)}\right)$ by the plotting position formula

$$
\begin{aligned}
& P_{i}=\frac{i}{n+1}, i=1,2, \ldots, n \\
& F\left(t_{(i)}\right)=\frac{i}{n+1}, i=1,2, \ldots, n
\end{aligned}
$$

Then

$$
F\left(t_{(1)}\right)=\frac{1}{n+1}
$$

From equations (2) and (7), we get

$$
\exp \left(-\alpha t_{(1)}{ }^{\beta} e^{\lambda t_{(1)}}\right)=1-\frac{1}{n+1}
$$
get

By taking the natural logarithm of equation (8), we

$$
\hat{\alpha}_{M M E}=\frac{-\operatorname{Ln}\left(1-\frac{1}{n+1}\right)}{t_{(1)}^{\beta} e^{\lambda t_{(1)}}}
$$

Where the symbol $\hat{\alpha}_{M M E}$ indicates the estimate of the scale parameter $\alpha$ by using MM method.

\section{Maximum Likelihood Estimator (MLE)}

The likelihood function for three-parameter Modified Weibull distribution (1) is [1].

$$
\begin{aligned}
L\left(\alpha, \beta, \lambda ; t_{1}, t_{2}, \ldots, t_{n}\right)= & \alpha^{n} \prod_{i=1}^{n}\left(\beta+\lambda t_{i}\right) \prod_{i=1}^{n} t_{i}^{\beta-1} \\
& \exp \sum_{i=1}^{n}\left(\lambda t_{i}-\alpha t_{i}{ }^{\beta} e^{\lambda t_{i}}\right)
\end{aligned}
$$

Taking the logarithm of the likelihood function, so we get the function

$$
\begin{aligned}
\ln L= & n \ln \alpha+\sum_{i=1}^{n} \ln \left(\beta+\lambda t_{i}\right)+ \\
& \left.(\beta-1) \sum_{i=1}^{n} \ln t_{i}+\sum_{i=1}^{n}\left(\lambda t_{i}-\alpha t_{i}{ }^{\beta} e^{\lambda t_{i}}\right)\right)
\end{aligned}
$$

The partial derivative of the log-likelihood function with respect to unknown parameters $\alpha$ is

$$
\frac{\partial \ln L}{\partial \alpha}=\frac{n}{\alpha}-\sum_{i=1}^{n} t_{i}^{\beta} e^{\lambda t_{i}}
$$

We place the partial derivative for log-likelihood with respect to $\alpha$ to zero as follows

$$
\begin{aligned}
& \frac{n}{\hat{\alpha}}-\sum_{i=1}^{n} t_{i}^{\beta} e^{\lambda t_{i}}=0 \\
& \hat{\alpha}_{M L E}=\frac{n}{\sum_{i=1}^{n} t_{i}^{\beta} e^{\lambda t_{i}}}
\end{aligned}
$$

Where the symbol $\hat{\alpha}_{M L E}$ indicates the estimate of the scale parameter $\alpha$ by using MLE method.

\section{Least Squares Estimator (LSE)}

The idea of this method is to minimize the sum of squared differences between observed sample values and the estimate expected values by linear approximation [6].

$$
\begin{aligned}
& \varepsilon_{i}=y_{i}-\beta_{0}-\beta_{1} x_{1}-\beta_{2} x_{2} \\
& \sum_{i=1}^{n} \varepsilon_{i}^{2}=\sum_{i=1}^{n}\left[y_{i}-\hat{y}_{i}\right]^{2} \\
& \sum_{i=1}^{n} \varepsilon_{i}^{2}=\sum_{i=1}^{n}\left[y_{i}-\hat{\beta}_{0}-\hat{\beta}_{1} x_{1}-\hat{\beta}_{2} x_{2}\right]^{2}
\end{aligned}
$$

By using the CDF of modified Weibull distribution (2) which are as follows

$$
1-\left[F\left(t_{i}\right)\right]=\exp \left(-\alpha t_{i}^{\beta} e^{\lambda t_{i}}\right)
$$

By taking the double logarithm of above equation getting

$\ln \left(-\ln \left[1-\left\{F\left(t_{i}\right)\right\}\right]\right)=\ln \alpha+\beta \ln t_{i}+\lambda t_{i}$

Comparing the above equation with the simple linear model

$Y=\beta_{0}+\beta_{1} x_{1}+\beta_{2} x_{2}+\varepsilon$

We get

$Y=\ln \left(-\ln \left[1-\left\{F\left(t_{i}\right)\right\}\right]\right), x_{1}=\ln t_{i}, x_{2}=t_{i}$,

$\beta_{0}=\ln \alpha, \beta_{1}=\beta, \beta_{2}=\lambda$.

$\varepsilon=\ln \left(-\ln \left[1-\left\{F\left(t_{i}\right)\right\}\right]\right)-\ln \alpha-\beta \ln t_{i}-\lambda t_{i}$

By taking the sum square of above equation for the two sides to reach

$\sum_{i=1}^{n} \varepsilon_{i}^{2}=\sum_{i=1}^{n}\left[\ln \left(-\ln \left[1-\left\{F\left(t_{i}\right)\right\}\right]\right)-\ln \alpha-\right.$
$\left.\beta \ln t_{i}-\lambda t_{i}\right]^{2}$

$$
\begin{gathered}
\frac{\partial\left(\sum_{i=1}^{n} \varepsilon_{i}^{2}\right)}{\partial \alpha}=-\frac{2}{\alpha} \sum_{i=1}^{n}\left[\ln \left(-\ln \left[1-\left\{F\left(t_{i}\right)\right\}\right]\right)\right. \\
\left.-\ln \alpha-\beta \ln t_{i}-\lambda t_{i}\right]
\end{gathered}
$$

We place the partial derivative $\sum_{i=1}^{n} \varepsilon_{i}^{2}$ to zero as follows 
$-\frac{2}{\alpha} \sum_{i=1}^{n}\left[\ln \left(-\ln \left[1-\left\{F\left(t_{i}\right)\right\}\right]\right)-\ln \alpha-\beta \ln t_{i}-\lambda t_{i}\right]=0$

$\hat{\alpha}_{L S E}=\exp \left(\frac{\sum_{i=1}^{n} \ln \left(-\ln \left[1-\left\{F\left(t_{i}\right)\right\}\right]\right)-\beta \sum_{i=1}^{n} \ln t_{i}-\lambda \sum_{i=1}^{n} t_{i}}{n}\right)$

Where the symbol $\hat{\alpha}_{L S E}$ indicates the estimate of the scale parameter $\alpha$ by using LSE Method and $F\left(t_{i}\right)$ is Empirical Distribution Functions which is [7].

$F\left(t_{i}\right)=\frac{i-0.5}{n}$

\section{White Estimator (WE)}

This method is mainly based on the reliability function of the distribution whose parameters are to be estimated and the formula of the function converted to a formula similar to the Linear Regression Equation, and its characteristics are to use its estimators as primary estimators for other estimation methods.

This method is applied to the modified Weibull distribution by taking the natural logarithm of both sides of the formula (3) we get the following formula

$$
-\ln R(x)=\alpha t^{\beta} e^{\lambda t}
$$

Comparing formula (26) with the following linear regression formula [8].

$$
\begin{aligned}
& z=\phi y+\varphi \\
& z=-\ln R(t), \phi=\alpha, y=\left(t^{\beta} e^{\lambda t}\right), \varphi=0 \\
& \hat{\phi}=\frac{\sum_{i=1}^{n}\left(y_{i}-\bar{y}\right)\left(z_{i}-\bar{z}\right)}{\sum_{i=1}^{n}\left(y_{i}-\bar{y}\right)^{2}}
\end{aligned}
$$

Where

$$
\bar{z}=\frac{\sum_{i=1}^{n} z_{i}}{n}=\frac{\sum_{i=1}^{n}-\ln R\left(t_{i}\right)}{n}
$$

And

$$
R\left(t_{i}\right)=1-F\left(t_{i}\right)=1-\frac{i-0.5}{n}
$$

Set $(\hat{\phi})$ in form (28) we obtain

$$
\hat{\alpha}_{W E}=\hat{\phi}
$$

Where the symbol $\hat{\alpha}_{W E}$ indicates the estimate of the scale parameter $\alpha$ by using WE Method.

\section{Monte Carlo Simulation}

The Monte Carlo simulation is using to compare the MSE for the scale parameter modified Weibull distributions with respect to MME, MLE, WE and OLSE methods, in this study we used the three models. The first model is $\alpha=0.1, \beta=0.2, \lambda=2$, the second model is $\alpha=0.05, \beta=0.2, \lambda=0.1$ and the third model is $\alpha=0.3, \beta=2, \lambda=0.2$.the probability density functions with this models are illustrated in Fig. 1

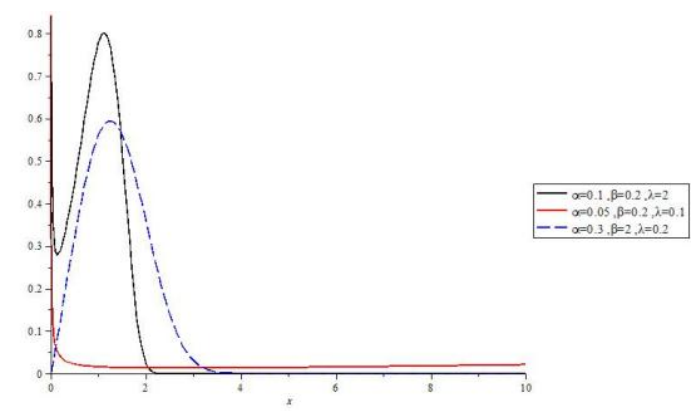

Fig.1. Different models shapes of pdf versus time

The MSE for any model in this Simulation is calculated using 24,800 simulated samples. All computations in this simulation are performed using MATLAB R2014a. We consider sample sizes $n=10$, 25, 100 and 200. We can generate random numbers from the modified Weibull distribution by using the inversion of the cumulative distribution function. And we replicate the data of experiment $\mathrm{N}$ times $(\mathrm{N}=200,500,1000,2000,2500)$ with sample size $\mathrm{n}$, the results of simulation presented in the following Tables. 


\begin{tabular}{|c|c|c|c|c|c|}
\hline \multirow{2}{*}{$\frac{\text { Cr. }}{\text { Meth. }}$} & \multicolumn{4}{|c|}{ MSE } & \multirow[b]{2}{*}{$\mathrm{N}$} \\
\hline & MME & MLE & WE & LSE & \\
\hline \multirow{5}{*}{10} & 2.383729236800643 & 0.001438063444104 & 0.002239672831175 & 0.002669346419856 & 200 \\
\hline & 33.648011790890713 & 0.001765433113177 & 0.003885925331741 & 0.003269032572172 & 500 \\
\hline & 21.252011566744255 & 0.001655171755509 & 0.004702689841331 & 0.002667558871810 & 1000 \\
\hline & 31.479130706661955 & 0.001602443785124 & 0.004870285091825 & 0.002507155344652 & 2000 \\
\hline & 23.075840594685047 & 0.001561226500369 & 0.006141199825043 & 0.002628589177462 & 2500 \\
\hline \multirow{5}{*}{25} & 6.033424239420221 & 0.000616354782930 & 0.000841312007086 & 0.001453861618977 & 200 \\
\hline & 4.507147322274402 & 0.000545467405829 & 0.001085909024811 & 0.000897824828307 & 500 \\
\hline & 19.206956103971507 & 0.000475412286613 & 0.001391672857426 & 0.000806102267597 & 1000 \\
\hline & 6.086150606366833 & 0.000473905174187 & 0.001444151975628 & 0.000862067567216 & 2000 \\
\hline & 19.350973297827583 & 0.000490254983197 & 0.001973022131112 & 0.000777758467862 & 2500 \\
\hline \multirow{5}{*}{100} & 3.210931476253547 & 0.000071962327029 & 0.000227684493831 & 0.000130169924409 & 200 \\
\hline & 1.454521076404451 & 0.000102951778319 & 0.000275130880454 & 0.000179175351622 & 500 \\
\hline & 10.616429106555705 & 0.000093776771464 & 0.000316419393898 & 0.000162374177441 & 1000 \\
\hline & 24.339393251617938 & 0.000098610585834 & 0.000342047813986 & 0.000159220442238 & 2000 \\
\hline & 99.336813474673278 & 0.000104208303217 & 0.000468051412648 & 0.000170623720589 & 2500 \\
\hline \multirow{5}{*}{200} & 2.214592525395552 & 0.000054626588130 & 0.000096813656970 & 0.000087189024627 & 200 \\
\hline & 5.975713459530112 & 0.000046840887403 & 0.000126351090577 & 0.000081744652007 & 500 \\
\hline & 20.413847579365985 & 0.000051966435075 & 0.000162230952909 & 0.000087498830131 & 1000 \\
\hline & 12.057602293630540 & 0.000050726119845 & 0.000175632920196 & 0.000084772316627 & 2000 \\
\hline & 9.972721383806443 & 0.000050804962080 & 0.000239815413910 & 0.000083709803411 & 2500 \\
\hline
\end{tabular}

Table-1. MSE of scale parameter $\alpha$ for the first model

\begin{tabular}{|c|c|c|c|c|c|}
\hline \multirow{2}{*}{$\begin{array}{c}\text { Cr. } \\
\text { Meth. }\end{array}$} & \multicolumn{4}{|c|}{ MSE } & \multirow[b]{2}{*}{$\mathrm{N}$} \\
\hline & MME & MLE & WE & LSE & \\
\hline \multirow{5}{*}{10} & 0.600528542955062 & 0.000362103793363 & 0.000682870997850 & 0.000657123460946 & 200 \\
\hline & 8.648731876098426 & 0.000574184732385 & 0.000912874105925 & 0.001075636703398 & 500 \\
\hline & 30.235692929472133 & 0.000729490743855 & 0.001093849682803 & 0.001255720751272 & 1000 \\
\hline & 23.147495988702524 & 0.000739364551927 & 0.001179560068047 & 0.001235487059464 & 2000 \\
\hline & 97.329825822911943 & 0.001036060095879 & 0.001675616166989 & 0.001677181513686 & 2500 \\
\hline \multirow{5}{*}{25} & 0.560890317361752 &.$\cdots$ ITVVITOKVTT. & 0.000952598840050 & 0.000240620231339 & 200 \\
\hline & 1.296706222958869 & 0.000173725581724 & 0.000247168907412 & 0.000296538842959 & 500 \\
\hline & 5.190199546287034 & 0.000202547441944 & 0.000318077071852 & 0.000342345089545 & 1000 \\
\hline & 24.519165732042783 & 0.000223687261805 & 0.000352924856757 & 0.000384973562539 & 2000 \\
\hline & 16.470487995151860 & 0.000121449654185 & 0.000487054189287 & 0.000208214775373 & 2500 \\
\hline \multirow{5}{*}{100} & 14.133282096560173 & 0.000025527706921 & 0.000042877140125 & 0.000039033745534 & 200 \\
\hline & 6.999907208554048 & 0.000035479752785 & 0.000065512298778 & 0.000057606322895 & 500 \\
\hline & 3.966748072195498 & 0.000045260998636 & 0.000079070454860 & 0.000073347671188 & 1000 \\
\hline & 438.7659200985143 & 0.00004785263240 & 0.000088558994225 & 0.000077234908500 & 2000 \\
\hline & 359.1210829682811 & 0.00006449266160 & 0.000118367166696 & 0.000103925437500 & 2500 \\
\hline \multirow{5}{*}{200} & 24.097332481215879 & 0.000013911561315 & 0.000024075051749 & 0.000023895975042 & 200 \\
\hline & 26.759433362973123 & 0.000018244664363 & 0.000036646460792 & 0.000031587280336 & 500 \\
\hline & 17.992704050140315 & 0.000020490848670 & 0.000039537033232 & 0.000034800882159 & 1000 \\
\hline & 531.3921693138716 & 0.00002270771970 & 0.000044770081252 & 0.000038863877600 & 2000 \\
\hline & 430.5269491736749 & 0.00003088326490 & 0.000060449409516 & 0.000051601324300 & 2500 \\
\hline
\end{tabular}

Table-2. MSE of scale parameter $\alpha$ for the second model 


\begin{tabular}{|c|c|c|c|c|c|}
\hline Cr. & \multicolumn{3}{|c|}{ MSE } & \\
\hline \multirow{3}{*}{ Meth. } & MME & MLE & WE & LSE & $\mathrm{N}$ \\
\hline \multirow{4}{*}{10} & 92.783635257522718 & 0.021375659212246 & 0.024583355922621 & 0.033511280002231 & 200 \\
\cline { 2 - 7 } & 900.8764969978296 & 0.026903098460800 & 0.032863467813329 & 0.041021218490000 & 500 \\
\cline { 2 - 7 } & 4032.351352442018 & 0.026240629047000 & 0.039378588580908 & 0.042246888000000 & 1000 \\
\cline { 2 - 7 } & 1249.939960709109 & 0.026092726897000 & 0.042464162449683 & 0.047452379245000 & 2000 \\
\cline { 2 - 7 } & 9905.778740941878 & 0.034568533836000 & 0.060322182011600 & 0.061281843026000 & 2500 \\
\hline \multirow{4}{*}{25} & 56.574535102180100 & 0.003309256212745 & 0.007029355824195 & 0.006213497462386 & 200 \\
\cline { 2 - 7 } & 469.6859933465812 & 0.004983251232800 & 0.008898080666838 & 0.089833525741000 & 500 \\
\cline { 2 - 7 } & 291.0073221553217 & 0.007531189153100 & 0.011450774586706 & 0.013178561010400 & 1000 \\
\cline { 2 - 7 } & 898.4793785900660 & 0.007865722885000 & 0.012705294843266 & 0.013474168028600 & 2000 \\
\cline { 2 - 7 } & 1279.291462242854 & 0.010626346911000 & 0.017533950814360 & 0.018584726738000 & 2500 \\
\hline \multirow{4}{*}{100} & 15.632592952906153 & 0.000977621525935 & 0.001686915251769 & 0.001557690728678 & 200 \\
\cline { 2 - 7 } & 27.297885386289721 & 0.001348569043347 & 0.002495441162018 & 0.002265631504168 & 500 \\
\cline { 2 - 7 } & 40.967280881829538 & 0.001650572281862 & 0.003081280689277 & 0.002644477362376 & 1000 \\
\cline { 2 - 7 } & 15945.93289070901 & 0.001736131620000 & 0.003215367422850 & 0.002805816320000 & 2000 \\
\cline { 2 - 7 } & 13172.30320540781 & 0.002322145640000 & 0.004331281405101 & 0.003760887680000 & 2500 \\
\hline \multirow{4}{*}{200} & 1500.818106616001 & 0.000511532437000 & 0.000851435380450 & 0.000873104024000 & 200 \\
\cline { 2 - 7 } & 6312.182944410619 & 0.000602154237100 & 0.001080440933797 & 0.001045570960600 & 500 \\
\cline { 2 - 7 } & 527.8395062493505 & 0.000724162434800 & 0.001370912560041 & 0.001256029543400 & 1000 \\
\cline { 2 - 7 } & 19061.14861102400 & 0.000813865600000 & 0.001592103002813 & 0.001388968840000 & 2000 \\
\cline { 2 - 7 } & 15444.74049969535 & 0.001104891550000 & $\cdot .002153481173084$ & 0.001837551020000 & 2500 \\
\hline
\end{tabular}

Table-3. MSE of scale parameter $\alpha$ for the third model

\section{The conclusion}

Note that we can make the following comments for the results in the above tables:

(1)The results of simulation presented in Table 1 is following conclusions may be summarized: the MLE is the best method and comes after LSE except when $\mathrm{n}=10,25$ with $\mathrm{N}=200$ according to the MSE criterion. (2)The results of simulation presented in Table 2 is following conclusions may be summarized: the MLE is the best method and comes after LSE except when $\mathrm{n}=10$ with $\mathrm{N}=500,1000,2000,2500$ and when $\mathrm{n}=25$ with $\mathrm{N}=500,1000,2000$ according to the MSE criterion.

(3)The results of simulation presented in Table 3 is following conclusions may be summarized: the MLE is the best method and comes after LSE except when $\mathrm{n}=10$ and when $\mathrm{n}=25$ with $\mathrm{N}=500,1000,2000,2500$ and when $n=200$ with $\mathrm{N}=200$, according to the MSE criterion.

\section{References}

[1]C.D. Lai, M. Xie and D.N. Murthy, "A modified Weibull distribution, IEEE Trans. Reliab. ", 52(2003), 33-37.

[2]P. Vasile, P. Eugenia and C. Alina, "Bayes estimators of modified Weibull distribution parameters using Lindley's approximation", WSEAS Transactions on Mathematics, 9(2010), 539-549.

[3]S.K. Upadhyaya, and A. Gupta, "A Bayes analysis of modified Weibull distribution via Markov chain Monte Carlo simulation", J. Statist. Comput. Simul.80 (2010), 241-254 .

[4]S.F. Ateya, "Estimation Under Modified Weibull Distribution Based on Right Censored Generalized Order Statistics", J. Applied Statistics, 40(2013), 2720-2734. 
[5]Cohen, A.C. and Whitten, B.J. "Modified Moment And Maximum Likelihood Estimators For Parameters Of Three Parameters Gamma Distribution", Commutation In Statistics, Simulation And Computation, (1982), 11,197-216.

[6]Ling, J., and Pan, J."A new Method for Selection of Population Distribution and Parameter Estimation", Reliability Engineering and System Safety, (1998), Vol. 60,pp. 247-255.
[7]Jasim, S.A. "Estimate Survival Function for the Brain Cancer Disease by using three Parameters Weibull Distribution", M.S.c . Thesis, Baghdad University, College of Education (Ibn Al-Hatham), (2010).

[8]Kadhem, Amori Hadi and Dulaimi, Muhammad Manaf, "Introduction to linear regression analysis", Ministry of Higher Education and Scientific Research, University of Baghdad, (1988).

\section{مقارنة بين أربع طرق لابيزية لتقدير معلمة القياس لتوزيع وييل المعدل باستخدام المحاكاة}

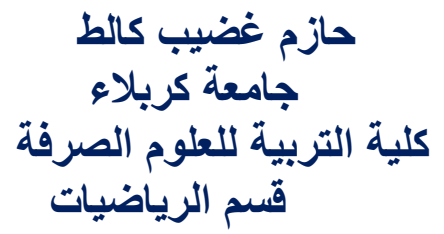

كلية التربية للعلوم الصرفة كرياءو

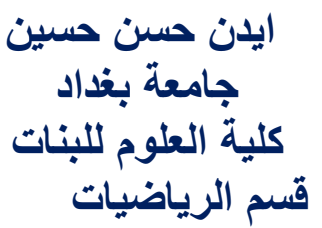

في هذه البحث ، تم الحصول على أربع طرق لتقدير معلمة المقياس لتوزيع ويبل المعدل باستخدام البيانات الكاملة، وهي

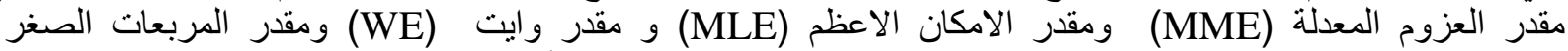
وقد استخدمت محاكاة مونت كارلو للمقارنة بين هذه المقدرات الأربعة وفقا لمعيار متوسط مربعات الخطأ (LSE)

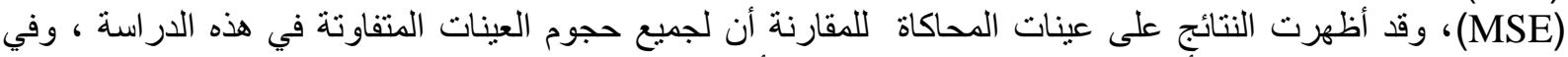

جميع الحالات عن الطرق الأربعة بان طريقة MLE هي الأفضل يتبعها طريقة OLSE ثم طريقة WE 